\title{
Immunohistochemistry for transcription factor T-Pit as a tool in diagnostics of corticotroph pituitary tumours
}

\author{
Olivera Casar-Borota ${ }^{1}\left[\right.$. Jens Bollerslev ${ }^{2} \cdot$ Fredrik Pontén $^{1}$
}

Published online: 21 February 2018

๑ Springer Science+Business Media, LLC, part of Springer Nature 2018

\section{To the Editor,}

We have read with interest the recent review on silent corticotroph pituitary tumours published in the Pituitary [1]. The review provides an update on pathogenesis, molecular biology and clinical behaviour of the silent corticotroph tumours, recognised by the 2017 WHO classification of pituitary tumours as a subtype with potentially more-aggressive behaviour.

In the introduction, the authors comment on immunohistochemistry with anti-T-Pit antibody that may result in reclassification of a subset of clinically non-functioning pituitary neuroendocrine tumours as silent corticotroph tumours. However, throughout the manuscript, the authors cite the older literature on the topic and fail to provide more recent literature citations with updated information regarding function and availability of a new T-Pit antibody.

Immunohistochemistry for transcription factor T-Pit is an essential tool in the diagnostics of corticotroph pituitary tumours with sparse or no ACTH immunolabeling. Thus, we believe that it is important to inform the readers that we recently presented a novel anti-T-Pit antibody as a reliable marker of corticotroph cell differentiation [2]. The antibody demonstrated distinct nuclear immunolabeling in tumour cells of corticotroph tumours, both hormone-producing and clinically silent. Immunohistochemistry using this new antibody resulted in re-classification of more than $50 \%$ of the tumours previously diagnosed as null-cell adenomas in a large and well-characterised cohort of pituitary neuroendocrine tumours. The importance of T-Pit immunohistochemistry, especially in corticotroph pituitary tumours with sparse or no ACTH-immunolabeling has also been commented on and illustrated by a figure in a recent review on non-functioning pituitary neuroendocrine tumours [3].

\section{Compliance with ethical standards}

Conflict of interest Fredrik Pontén is a co-founder of Atlas Antibodies. The other authors have no conflicts of interest to disclose.

\section{References}

1. Ben-Shlomo A, Cooper O (2018) Silent corticotroph adenomas. Pituitary. https://doi.org/10.1007/s11102-018-0864-8

2. Sjöstedt E, Bollerslev J, Mulder J, Lindskog C, Pontén F, CasarBorota O (2017) A specific antibody to detect transcription factor T-Pit: a reliable marker of corticotroph cell differentiation and a tool to improve the classification of pituitary neuroendocrine tumours. Acta Neuropathol 134:675-677

3. Manojlovic-Gacic E, Engström BE, Casar-Borota O (2017) Histopathological classification of non-functioning pituitary neuroendocrine tumors. Pituitary. https://doi.org/10.1007/s1110 2-017-0855-1
Olivera Casar-Borota

olivera.casar-borota@igp.uu.se

Uppsala University, Uppsala, Sweden

2 University of Oslo, Oslo, Norway 